\title{
Research long-term fluctuations in winter temperatures. Methods for analyzing the intensities and synchronicities of fluctuations in fuel consumption
}

\author{
Ivan Khazheev ${ }^{1, *}$ \\ ${ }^{1}$ Melentiev Energy Systems Institute SB RAS, Department of Applied Mathematics, 664033 Irkutsk, Lermontov st., 130, Russia
}

\begin{abstract}
The article deals with the problem of assessing the deviations of the meteorological characteristics of the heating season. The usual method of calculating the relative deviations of indicators from the arithmetic mean is not suitable. A requirement is imposed on the "new" averages and estimates of deviations of meteorological characteristics, which follows from their properties: the duration multiplied by the average daily temperature difference for the heating period should be equal to the integral temperature difference. The application of this property to the means and to the very estimates of the deviations of meteorological characteristics makes it difficult to determine the latter. A method is being developed to determine the intensities of fluctuations in the meteorological characteristics of the heating season. Intensities show how much, on average, the duration, average daily and integral temperature differences for the heating period can deviate from the average expected level. A technique is being developed for reflecting synchronous and asynchronous fluctuations in deviations of meteorological characteristics, and the contributions of these fluctuations are determined.
\end{abstract}

\section{Introduction}

Due to the long heating period and low winter temperatures, the study of climatic parameters of the heating period is of greater importance for Russia. These are the outdoor temperature, wind speed, air humidity, the intensity of solar radiation. The values of these indicators are used to assess the winter season of the region in question.

Usually the heating period of the region in question is determined by its duration and ambient air temperatures. In coastal areas, an additional cold load can be caused by relative humidity.

Let $\tau=1,2, \ldots, T$ are the numbers of the studied heating periods, $T$ - the number of heating periods. $L_{\tau}$ denote the duration of the heating period $\tau$. The values $i=1,2, \ldots, L_{\tau}$ as the sequence numbers correspond to day heating period $\tau$.

Each heating season starts in the autumn of one calendar year and ends in the spring of the following calendar year. Therefore, each ordinal number of the heating period corresponds to two calendar years. So, the last heating period began in 2020 and ended in 2021 .

The article uses the estimated duration of the heating period. To determine it, the following rule is used: the heating period begins if, for five consecutive days, the average daily temperature of atmospheric air is below 8 degrees Celsius. The heating period is considered over if the air temperature is above 8 degrees Celsius for five consecutive days. This formal rule is usually followed by heating systems in settlements.

In solving many problems associated with the heat supply of buildings, an indicator of the integral temperature difference inside and outside the building for the heating period is used. For the considered settlement or district, the indicator of the integral temperature difference is calculated by the formula:

$$
B_{\tau}=\sum_{i=1}\left(t_{n}-t_{\tau i}\right), \quad i=1,2, \ldots, L_{\tau}, \quad \tau=1,2, \ldots, T
$$

Here, the value $t_{t i}$ is the average daily temperature of the air on the day $i$ of the heating period $\tau$. The indicator $B_{\tau}$ is determined on the basis of meteorological observations.

The value $t_{n}$ is the specified normative value of the air temperature inside the building. Depending on the purpose of the building, there may be different values of normative temperatures. For residential premises, a temperature of 20 degrees Celsius can be used for the standard, for children's institutions - 24 degrees Celsius, for office premises - 18 degrees Celsius. Whereas in production rooms and warehouses, the normative temperature can be 14 degrees Celsius. In the calculations presented in this article, a standard value of 18 degrees Celsius was used.

* Corresponding author: ivan-khazheev@yandex.ru 
The use of the indicator of the integral temperature difference inside and outside the building for the heating period (1) is based on the law of thermal conductivity: the loss of thermal energy through the fencing, in the construction of buildings is proportional to the difference in temperature inside and outside the building. Therefore, the ratio of the integral temperature difference in different settlements or in different years for the same settlements reflect the ratio of heat energy consumption for heating and, as a result, fuel consumption for heat for heating.

The integral temperature difference is in practice applicable for calculating the optimal building structures at which heat losses are minimized.

The integral temperature difference inside and outside the building can be represented as a multiplication of two other indicators: the duration of the heating period and the average daily temperature difference inside and outside the building during the heating period.

$$
B_{\tau}=L_{\tau} N_{\tau}, \tau=1,2, \ldots, T
$$

where $N_{\tau}, \quad \tau=1,2, \ldots, T$ is the average daily temperature difference for the heating period inside and outside the building.

Traditionally, to assess deviations of indicators, relative deviations from the arithmetic mean values are used:

- $\quad$ for the integral temperature difference inside and outside the building for the heating period:

$$
b_{\tau A}=B_{\tau} / B_{A} \text {, }
$$

- for the duration of the heating period:

$$
l_{\tau A}=L_{\tau} / L_{A} \text {, }
$$

- for the average daily temperature difference during the heating period:

$$
n_{\tau A}=N_{\tau} / N_{A}
$$

where $B_{A}, L_{A}, N_{A}$ - the arithmetic mean values of the indicators $B_{\tau}, L_{\tau}, N_{\tau} \tau=1,2, \ldots, T$.

The multiplication of the arithmetic means of the duration and the daily temperature difference inside and outside the building during the heating period is not equal to the arithmetic mean integral temperature difference:

$$
L_{A} \cdot N_{A} \neq B_{A} \text {. }
$$

Consequently, the multiplication of the relative deviations of the duration and the average daily temperature difference for the heating period from the arithmetic means is not equal to the relative deviation of the integral temperature difference:

$$
l_{\tau A} \cdot n_{\tau A} \neq b_{\tau A} \text {. }
$$

The ability to represent the average and estimate the deviations of the integral temperature difference in the form of multiplying the corresponding duration and the average daily temperature difference for the heating period becomes the main requirement for the average and for the estimates of deviations.

\section{Intensities of fluctuations in the meteorological characteristics of the heating period}

In addition to the arithmetic means, can use the geometric means $B_{G}, L_{G}, N_{G}$. For geometric averages, the multiplication of the average for the observation period of the duration and the average daily temperature difference for the heating period is equal to the average integral temperature difference:

$$
L_{G} \cdot N_{G}=B_{G} \text {. }
$$

similarly for relative deviations:

$$
l_{\tau G} \cdot n_{\tau G}=b_{\tau G}
$$

where $b_{\tau G}, l_{\tau G}, n_{\tau G}$ are the relative deviations $B_{\tau}, L_{\tau}, N_{\tau} \tau$ $=1,2, \ldots, T$ from the geometric means $B_{G}, L_{G}, N_{G}$.

When working with geometric means, it is necessary to take into account their deviations from the arithmetic mean: for any unequal values of the averaged indicator, the geometric mean is always less than the arithmetic mean. However, from numerical calculations, we find that the geometric mean values deviate from the arithmetic mean insignificantly, up to $1 \%$.

Geometric averages are not the only method for averaging the values of climatic characteristics, the use of which ensures the equality of the multiplications of the average and relative deviations of the duration and the average daily temperature difference for the heating period to the corresponding indicators of the integral temperature difference. There are other, more complex, multi-stage methods, but in this article we will not focus on such methods, we will use geometric mean for calculations.

Let represent the relative deviations of the meteorological characteristics of the heating season on a logarithmic scale: duration, average daily and integral temperature differences for the heating season

Introduce the intensity of fluctuations into the duration, average daily and integral temperature drops during the heating period $-I_{b}, l_{l}, I_{n}$.

$$
\begin{aligned}
& I_{b}=\exp \left(\sum_{\tau=1,2, \ldots, T}\left|\ln b_{\tau G}\right|\right) / T, \\
& I_{l}=\exp \left(\sum_{\tau=1,2, \ldots, T}\left|\ln l_{\tau G}\right|\right) / T, \\
& I_{n}=\exp \left(\sum_{\tau=1,2, \ldots, T}\left|\ln n_{\tau G}\right|\right) / T .
\end{aligned}
$$

The intensities of fluctuations show how, on average, the indicators of the heating period can deviate from the average expected level.

\section{Synchronous and asynchronous fluctuations in the duration and average daily temperature difference during the heating period}

Let introduce the sign function of the relative deviations:

$$
\mu(\alpha)=\left\{\begin{array}{l}
-1, \alpha<0, \\
1, \alpha \geq 0 .
\end{array}\right.
$$

Introduce the sets of numbers of heating periods:

$$
\begin{aligned}
& K_{L}{ }^{S}=\left\{t: \mu\left(l_{\tau G}\right) \cdot \mu\left(b_{\tau G}\right) \geq 0,\right. \\
& K_{L}{ }^{A}=\left\{t: \mu\left(l_{\tau G}\right) \cdot \mu\left(b_{\tau G}\right)<0,\right. \\
& K_{N}{ }^{S}=\left\{t: \mu\left(n_{\tau G}\right) \cdot \mu\left(b_{\tau G}\right) \geq 0,\right. \\
& K_{N}{ }^{A}=\left\{t: \mu\left(n_{\tau G}\right) \cdot \mu\left(b_{\tau G}\right)<0,\right.
\end{aligned}
$$

where $K_{L}^{S}, K_{N}^{S}$ are sets of numbers of heating periods, when the duration and the average daily temperature difference deviate unidirectionally, synchronously with the integral temperature difference; $K_{L}{ }^{A}, K_{N}{ }^{A}$ are sets of heating periods with multidirectional, asynchronous changes in the duration and average daily temperature difference from changes in the integral temperature difference. 
Calculate the intensities of synchronous $\left(I_{l}^{S}, I_{n}^{S}\right)$ and asynchronous $\left(I^{A}, I_{n}{ }^{A}\right)$ fluctuations in the duration and average daily temperature difference for the heating period based on these sets $K_{L}^{S}, K_{N}^{S}, K_{L}^{A}, K_{N}^{A}$.

Note that for the intensities of fluctuations in the duration and average daily temperature difference, the transitivity properties are fulfilled:

$$
\begin{aligned}
& I_{l}=I_{l}^{S} \cdot I_{l}^{A}, \\
& I_{n}=I_{n}{ }^{S} \cdot I_{n}{ }^{A} .
\end{aligned}
$$

The contributions of synchronous $\delta_{l}{ }^{S}$ and asynchronous $\delta_{l}^{A}$ fluctuations to the intensity of fluctuations in the duration of the heating period are determined by the formulas:

$$
\begin{aligned}
& \delta_{l}{ }^{S}=\ln I_{l}{ }^{S} / \ln I_{l}, \\
& \delta_{l}^{A}=\ln I_{l}^{A} / \ln I_{l} .
\end{aligned}
$$

Similarly, the contributions of synchronous $\delta_{n}{ }^{S}$ and asynchronous $\delta_{n}{ }^{A}$ fluctuations in the intensity of fluctuations in the average daily temperature difference for the heating period are determined by the formulas:

$$
\begin{aligned}
& \delta_{n}{ }^{S}=\ln I_{n}{ }^{S} / \ln I_{n}, \\
& \delta_{n}{ }^{A}=\ln I_{n}{ }^{A} / \ln I_{n} .
\end{aligned}
$$

Determine the contributions of synchronous $\delta_{L}{ }^{S}, \delta_{N} S$ and asynchronous $\delta_{L}{ }^{A}, \delta_{N}{ }^{A}$ fluctuations in the duration and average daily temperature difference in the intensity of fluctuations in the integral temperature difference:

$$
\begin{aligned}
& \delta_{L}{ }^{S}=\ln I_{L}{ }^{S} / \ln I_{b}, \\
& \delta_{L}{ }^{A}=\ln I_{L}{ }^{A} / \ln I_{b}, \\
& \delta_{N}{ }^{S}=\ln I_{N} S / \ln I_{b}, \\
& \delta_{N}{ }^{A}=\ln I_{N}{ }^{A} / \ln I_{b} .
\end{aligned}
$$

By combining the contributions of synchronous and asynchronous oscillations in the deviations of the integral temperature difference, it is possible to estimate the total contributions of the duration and the average daily temperature difference, the contributions of synchronous and asynchronous oscillations to the intensity of the oscillations of the integral temperature difference.

\section{Calculations}

Table 1 shows the intensity of fluctuations in duration, average daily and integral temperature differences for the heating period from long-term averages.

Table 1. Meteorological characteristics of fluctuations intensities of the heating period

\begin{tabular}{|c|c|c|c|c|}
\hline Index & Yakutsk & Irkutsk & Moscow & Simferopol \\
\hline $\boldsymbol{I}_{\boldsymbol{l}}$ & $\mathbf{1 , 0 2 4}$ & $\mathbf{1 , 0 2 7}$ & $\mathbf{1 , 0 4 7}$ & $\mathbf{1 , 1 5 1}$ \\
\hline$I_{l}{ }^{S}$ & 1,018 & 1,022 & 1,040 & 1,149 \\
\hline $\boldsymbol{I}^{A}$ & 1,006 & 1,005 & 1,006 & 1,001 \\
\hline $\boldsymbol{I}_{\boldsymbol{n}}$ & $\mathbf{1 , 0 3 3}$ & $\mathbf{1 , 0 3 8}$ & $\mathbf{1 , 0 5 3}$ & $\mathbf{1 , 0 4 5}$ \\
\hline $\boldsymbol{I}_{n}{ }^{S}$ & 1,029 & 1,035 & 1,049 & 1,038 \\
\hline $\boldsymbol{I}_{n}{ }^{A}$ & 1,004 & 1,003 & 1,004 & 1,008 \\
\hline $\boldsymbol{I}_{\boldsymbol{b}}$ & $\mathbf{1 , 0 3 8}$ & $\mathbf{1 , 0 5 0}$ & $\mathbf{1 , 0 7 9}$ & $\mathbf{1 , 1 8 2}$ \\
\hline
\end{tabular}

The data presented in Table 1 can be interpreted as follows. For the city of Yakutsk, the average value of fluctuations in the integral temperature difference from the long-term average is $103.8 \%$, that is, the deviation is $3.8 \%$, according to the duration of the heating period $2.4 \%$, according to the average daily temperature difference - $3.3 \%$. At the same time, the value of synchronous and asynchronous deviations of the duration of the heating period is $1.8 \%$ and $0.6 \%$, respectively, for the average daily temperature difference for the heating

\begin{tabular}{|c|c|c|c|c|}
\hline Index & Yakutsk & Irkutsk & Moscow & Simferopol \\
\hline \multicolumn{5}{|c|}{$\begin{array}{l}\text { Contributions of synchronous and asynchronous } \\
\text { fluctuations in the deviations of the duration of the heating } \\
\text { period }\end{array}$} \\
\hline$\delta_{l}^{S}$ & 76,6 & 82,1 & 86,2 & 99,0 \\
\hline$\delta_{l}^{A}$ & 23,4 & 17,9 & 13,8 & 1,0 \\
\hline \multicolumn{5}{|c|}{$\begin{array}{l}\text { Contributions of synchronous and asynchronous } \\
\text { fluctuations in deviations of the average daily temperature } \\
\text { difference inside and outside the building during the heating } \\
\text { period }\end{array}$} \\
\hline$\delta_{n}{ }^{S}$ & 87,5 & 92,0 & 91,7 & 82,9 \\
\hline$\delta_{n}{ }^{A}$ & 12,5 & 8,0 & 8,3 & 17,1 \\
\hline
\end{tabular}
period $-2.9 \%$ and $0.4 \%$, respectively.

Tables 2,3 show the contributions of synchronous and asynchronous fluctuations in the duration and average daily temperature difference for the heating period, as well as their contributions to the variations in the integral temperature difference.

Table 2. Relative weights of synchronous and asynchronous fluctuations in the deviations of the duration and average daily temperature difference

Table 3. Relative weights of synchronous and asynchronous fluctuations in the deviations of the integral temperature difference

\begin{tabular}{|c|c|c|c|c|}
\hline Index & Yakutsk & Irkutsk & Moscow & Simferopol \\
\hline$\delta_{L}{ }^{S}$ & 49,3 & 44,9 & 51,9 & 83,2 \\
\hline$\delta_{L}{ }^{A}$ & $-15,0$ & $-9,8$ & $-8,3$ & $-0,8$ \\
\hline$\delta_{N}{ }^{S}$ & 76,7 & 71,0 & 62,1 & 22,2 \\
\hline$\delta_{N}{ }^{A}$ & $-11,0$ & $-6,1$ & $-5,7$ & $-4,6$ \\
\hline
\end{tabular}

Calculate contributions of synchronous and asynchronous fluctuations, contributions of the duration and the average daily temperature difference in the deviations of the integral temperature difference (Fig. $1,2)$.

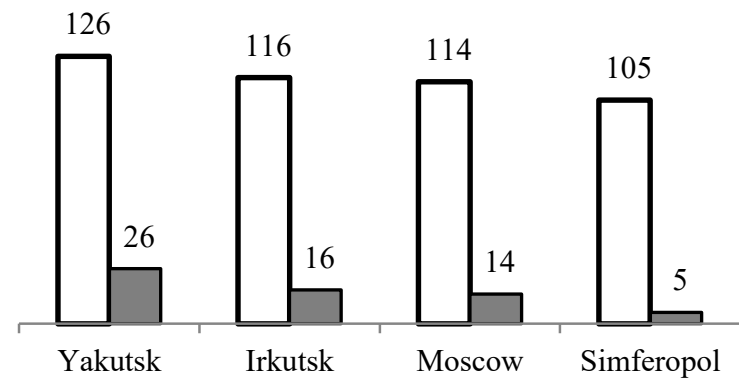


Fig. 1. Contributions of synchronous and asynchronous fluctuations in the deviations of the integral temperature difference

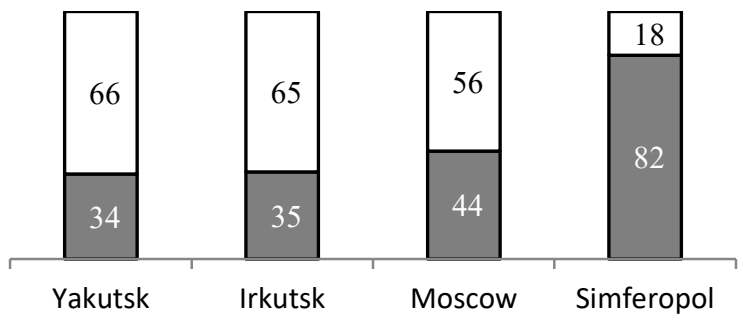

口Contributions of the average daily temperature difference in the deviations of the integral temperature difference

口Contributions of the duration in the deviations of the integral temperature difference

Fig. 2. Contributions of the duration and the average daily temperature difference in the deviations of the integral temperature difference

According to Fig. 1 and 2, as the integral temperature difference decreases, the contribution of asynchronous oscillations decreases and the contribution of deviations in the duration of the heating period increases.

The results obtained can be interpreted as follows:

1) during the transition from cold regions to warm ones, the contribution of the asynchronous component of deviations decreases;

For warmer regions, a change in the meteorological characteristics of a heating year: duration or temperatures - with a higher probability we will lead to a synchronous change in the integral temperature difference.

2 ) with the transition from cold to warm regions, the contribution of changes in the duration of the heating period increases.

For warmer regions, the duration of the heating period plays an increasing role in the deviations of the integral temperature difference; for Simferopol, up to $82 \%$ is explained by the variation in duration.

\section{Conclusions}

The problem of assessing the deviations of meteorological characteristics of the heating period is investigated. The use of geometric averages satisfies the requirement: the multiplication of the average for the observation period of the duration and the average daily temperature difference for the heating period coincide with the average integral temperature difference. In this case, the geometric mean values deviate from the arithmetic mean insignificantly, up to $1 \%$.

A method has been developed to determine the intensities of fluctuations in the meteorological characteristics of the heating season. Intensities show how much, on average, the duration, average daily and integral temperature differences for the heating period can deviate from the average expected level. A technique has been developed for taking into account synchronous and asynchronous oscillations in deviations of meteorological characteristics. Using the logarithmic method, the contributions of these fluctuations in the deviations of the duration and average daily temperature difference for the heating period, as well as in the deviations of the integral temperature difference, were determined.

Numerical calculations were made for the cities of Yakutsk, Irkutsk, Moscow and Simferopol, the following conclusions were obtained. For warmer regions, a change in duration or temperature is more likely to lead to a synchronous change in the integral temperature difference than for colder regions. At the same time, for warmer regions, an increasing role in the deviations of the integral temperature difference is played by the duration of the heating season, so for Simferopol, up to $82 \%$ is explained by the variation in duration.

The research was carried out with the financial support of the Russian Foundation for Basic Research (Grant № 19-07-00322).

\section{References}

1. M. Velikanov, A. Nekrasov, A. Reznikovskij, Proceedings of Academy of Sciences. Power Engeneering and Transport, 2, 151-157 (1988).

2. V. Zorkaltcev. Long-term temperature variations and impact on the economy and energy (Geo, Novosibirsk, 2017).

3. V. Zorkaltcev. Fluctuations in fuel demand for heating in the economic regions of the USSR (ESI SB RAS, Irkutsk, 1998).

4. V. Zorkaltcev, I. Mokryi, I. Khazheev. Long-term variations in winter temperatures in the regions of Russia and their impact on the reliability of power supply. Energy policy, 4, 81-91 (2017).

5. I. Khazheev. How does climate affect the economy? Eco, 7, 147-162 (2015).

6. I. Khazheev. How do you know when you meet? About predicting winter by the date of its beginning. Eco, 4, 124-133 (2017).

7. I. Khazheev. Research of fluctuations in the demand for energy resources for heating based on long-term meteorological data. System research in power engineering, 45, 144-151 (2015).

8. L. Khrilev. On the influence of the climatic factor on the perspective installation of the fuel and energy balance. Teploenergetika, 2, 16-20 (1966).

9. L. Khrilev. District heating and fuel and energy complex. (Nauka, Novosibirsk, 1979).

10. Yu. Rudenko. Methodological issues in the study of the reliability of large power systems. Proceedings of Academy of Sciences of the USSR. Energy and transport, 1, 7-24 (1976).

11. Russian Hydrometeorological Portal All-Russian Research Institute of Hydrometeorological Information. URL: http://aisori.meteo.ru/ClimateR. 